\title{
Flux Maximizing Geometric Flows
}

\author{
Alexander Vasilevskiy*
}

\author{
Kaleem Siddiqi ${ }^{\dagger}$
}

\begin{abstract}
Several geometric active contour models have been proposed for segmentation in computer vision. The essential idea is to evolve a curve (in 2D) or a surface (in $3 D$ ) under constraints from image forces so that it clings to features of interest in an intensity image. Recent variations on this theme take into account properties of enclosed regions and allow for multiple curves or surfaces to be simultaneously represented. However, it is not clear how to apply these techniques to images of low contrast elongated structures, such as those of blood vessels. To address this problem we derive the gradient flow which maximizes the rate of increase of flux of an auxiliary vector field through a curve or surface. The calculation leads to a simple and elegant interpretation which is essentially parameter free. We illustrate its advantages with level-set based segmentations of $2 D$ and $3 D$ MRA images of blood vessels.
\end{abstract}

\section{Introduction}

Level-set based numerical methods for hyperbolic conservation laws were developed by Osher and Sethian [14] for curvature-dependent flame propagation, and were introduced to the computer vision community for shape analysis by Kimia et al [9]. Such models were later adapted to the problem of shape segmentation independently by Caselles et al. [3] and Malladi et al. [13]. Here the essential idea was to halt a moving curve or surface in the presence of intensity edges by multiplying the evolution equation with an image gradient based stopping potential. This formulation led to a powerful new active contour model which had the advantage over classical snakes [7] that changes in topology due to the splitting and merging of multiple contours were handled in a natural way. These flows were later given formal motivation as well as unified with the classical energy minimization formulations through several independent investigations $[4,8,16,17]$.

Recently there have been other advances in the use of geometric flows in computer vision. First, a number of

\footnotetext{
${ }^{*}$ School of Computer Science \& Center for Intelligent Machines McGill University, Montréal, PQ, Canada H3A 2A7

†School of Computer Science \& Center for Intelligent Machines McGill University, Montréal, PQ, Canada H3A 2A7
}

researchers have proposed flows which take into account the statistics of the enclosed regions [15, 21]. Further developments include multi-phase motions [5] and the use of an external force field based on a diffused gradient of an edge map [20]. Second, Lorigo et al. have proposed the use of active contours with co-dimension 2 (curves in 3D) [12] to address the recovery of low contrast elongated structures. Here the flow is regularized by a term proportional to the curvature of a 3D curve, based on the level set theory for mean curvature evolution of surfaces of arbitrary co-dimension [1]. The solution can be interpreted as a gradient flow which minimizes a particular energy functional. However, the derived flow is later modified with a heuristic multiplicative term [12].

In this paper we seek an alternate approach to segmenting elongated structures, such as blood vessels in MR angiography (MRA) images. The development is motivated by the observation that blood flows in the direction of vessels and that brightness in MRA images is proportional to the magnitude of the blood flow velocity. This leads to the constraint that in the vicinity of blood vessel boundaries, the gradient vector field of an MRA image should be locally orthogonal to them. Thus, a natural principle to use towards the recovery of these boundaries is to maximize the flux of the gradient vector field through an evolving curve (in 2D) or surface (in 3D). The main contribution of this paper is the formulation and derivation of this flux maximizing gradient flow and its application to the segmentation of blood vessels.

\section{Flux Maximizing Flows}

Let $\mathcal{C}=\mathcal{C}(p, t)$ be a smooth family of closed curves evolving in the plane. Here $t$ parametrizes the family and $p$ the given curve. Without loss of generality we shall assume that $0 \leq p \leq 1$, i.e., that $\mathcal{C}(0, t)=\mathcal{C}(1, t)$. We shall also assume that the first derivatives exist and that $\mathcal{C}^{\prime}(0, t)=$ $\mathcal{C}^{\prime}(1, t)$. The unit tangent $\mathcal{T}$ and the unit inward normal $\mathcal{N}$ to $\mathcal{C}$ are given by

$\mathcal{T}=\frac{\left(\begin{array}{c}x_{p} \\ y_{p}\end{array}\right)}{\left\|\mathcal{C}_{p}\right\|}=\left(\begin{array}{c}x_{s} \\ y_{s}\end{array}\right) ; \mathcal{N}=\frac{\left(\begin{array}{c}-y_{p} \\ x_{p}\end{array}\right)}{\left\|\mathcal{C}_{p}\right\|}=\left(\begin{array}{c}-y_{s} \\ x_{s}\end{array}\right)$ 
where $s$ is the arc-length parametrization of the curve. Now consider a vector field $\mathcal{V}=\left(V_{1}(x, y), V_{2}(x, y)\right)$ defined for each point $(x, y)$ in $\mathcal{R}^{2}$. The total inward flux of the vector field through the curve is given by the contour integral

$$
\operatorname{Flux}(t)=\int_{0}^{1}\langle\mathcal{V}, \mathcal{N}\rangle\left\|\mathcal{C}_{p}\right\| d p=\int_{0}^{L(t)}\langle\mathcal{V}, \mathcal{N}\rangle d s
$$

where $L(t)$ is the Euclidean length of the curve. The circulation of the vector field along the curve is defined in an analogous fashion as

$$
\operatorname{Circ}(t)=\int_{0}^{1}\langle\mathcal{V}, \mathcal{T}\rangle\left\|\mathcal{C}_{p}\right\| d p=\int_{0}^{L(t)}\langle\mathcal{V}, \mathcal{T}\rangle d s
$$

The main theoretical result of this paper is the following theorem

Theorem 1 The direction in which the inward flux of the vector field $\mathcal{V}$ through the curve $\mathcal{C}$ is increasing most rapidly is given by $\frac{\partial \mathcal{C}}{\partial t}=\operatorname{div}(\mathcal{V}) \mathcal{N}$.

In other words, the gradient flow which maximizes the rate of increase of the total inward flux is obtained by moving each point of the curve in the direction of the inward normal by an amount proportional to the divergence of the vector field. As we shall later see, this result can be exploited to recover low contrast elongated structures such as blood vessels in MRA images.

Proof: Define the perpendicular to a vector $\mathcal{W}=(a, b)$ as $\mathcal{W}^{\perp}=(-b, a)$. The following properties hold:

$$
\begin{gathered}
\left\langle\mathcal{U}, \mathcal{W}^{\perp}\right\rangle=-\left\langle\mathcal{U}^{\perp}, \mathcal{W}\right\rangle \\
\left\langle\mathcal{U}^{\perp}, \mathcal{W}^{\perp}\right\rangle=\langle\mathcal{U}, \mathcal{W}\rangle .
\end{gathered}
$$

We now compute the first variation of the flux functional with respect to $t$

$$
\operatorname{Flux}^{\prime}(t)=\underbrace{\int_{0}^{L(t)}\left\langle\mathcal{V}_{t}, \mathcal{N}\right\rangle d s}_{I_{1}}+\underbrace{\int_{0}^{L(t)}\left\langle\mathcal{V}, \mathcal{N}_{t}\right\rangle d s}_{I_{2}}
$$

With

$$
\begin{gathered}
\mathcal{V}_{t}=\left(\frac{\partial V_{1}}{\partial t}, \frac{\partial V_{2}}{\partial t}\right)=\left(\left\langle\nabla V_{1}, \mathcal{C}_{t}\right\rangle,\left\langle\nabla V_{2}, \mathcal{C}_{t}\right\rangle\right) \\
I_{1}=\int_{0}^{L(t)}\left\langle\mathcal{C}_{t}, x_{s} \nabla V_{2}-y_{s} \nabla V_{1}\right\rangle d s .
\end{gathered}
$$

For $I_{2}$ we first switch to parametrization by $p$ :

$$
I_{2}=\int_{0}^{L(t)}\left\langle\mathcal{V}, \mathcal{N}_{t}\right\rangle d s=\int_{0}^{1}\left\langle\mathcal{V},\left(\begin{array}{c}
-y_{p t} \\
x_{p t}
\end{array}\right)\right\rangle d p
$$

Now, using integration by parts

$$
I_{2}=\underbrace{\left.\left\langle\mathcal{V},\left(\begin{array}{c}
-y_{t} \\
x_{t}
\end{array}\right)\right\rangle\right]_{0}^{1}}_{\text {equals } 0}-\int_{0}^{1}\left\langle\left(\begin{array}{c}
-y_{t} \\
x_{t}
\end{array}\right), \mathcal{V}_{p}\right\rangle d p
$$

Using the properties of scalar products in Eq. (2) and the fact that

$$
\mathcal{V}_{p}=\left(\frac{\partial V_{1}}{\partial p}, \frac{\partial V_{2}}{\partial p}\right)=\left(\left\langle\nabla V_{1}, \mathcal{C}_{p}\right\rangle,\left\langle\nabla V_{2}, \mathcal{C}_{p}\right\rangle\right)
$$

we can rewrite $I_{2}$ as follows

$$
\begin{gathered}
I_{2}=\int_{0}^{1}\left\langle\left(\begin{array}{c}
x_{t} \\
y_{t}
\end{array}\right), \mathcal{V}_{p}{ }^{\perp}\right\rangle d p \\
=\int_{0}^{1}\left\langle\mathcal{C}_{t},\left(\begin{array}{c}
-\left\langle\nabla V_{2}, \mathcal{C}_{p}\right\rangle \\
\left\langle\nabla V_{1}, \mathcal{C}_{p}\right\rangle
\end{array}\right)\right\rangle d p .
\end{gathered}
$$

Switching to arc-length parametrization

$$
I_{2}=\int_{0}^{L(t)}\left\langle\mathcal{C}_{t},\left(\begin{array}{c}
-\left\langle\nabla V_{2}, \mathcal{T}\right\rangle \\
\left\langle\nabla V_{1}, \mathcal{T}\right\rangle
\end{array}\right)\right\rangle d s
$$

Combining $I_{1}$ and $I_{2}$, the first variation of the flux is

$$
\int_{0}^{L(t)}\left\langle\mathcal{C}_{t}, x_{s} \nabla V_{2}-y_{s} \nabla V_{1}+\left(\begin{array}{c}
-\left\langle\nabla V_{2}, \mathcal{T}\right\rangle \\
\left\langle\nabla V_{1}, \mathcal{T}\right\rangle
\end{array}\right)\right\rangle d s
$$

Thus, for the flux to increase as fast as possible, the two vectors should be made parallel:

$$
\mathcal{C}_{t}=x_{s} \nabla V_{2}-y_{s} \nabla V_{1}+\left(\begin{array}{c}
-\left\langle\nabla V_{2}, \mathcal{T}\right\rangle \\
\left\langle\nabla V_{1}, \mathcal{T}\right\rangle
\end{array}\right) .
$$

Decomposing the above three vectors in the Frenet frame $\{\mathcal{T}, \mathcal{N}\}$, dropping the tangential terms (which affect only the parametrization of the curve) and making use of the properties of scalar products

$$
\begin{array}{r}
\mathcal{C}_{t}=\left\{x_{s}\left\langle\nabla V_{2}, \mathcal{N}\right\rangle-y_{s}\left\langle\nabla V_{1}, \mathcal{N}\right\rangle\right. \\
\left.+\left\langle\left(\begin{array}{c}
-\left\langle\nabla V_{2}{ }^{\perp}, \mathcal{N}\right\rangle \\
\left\langle\nabla V_{1}{ }^{\perp}, \mathcal{N}\right\rangle
\end{array}\right), \mathcal{N}\right\rangle\right\} \mathcal{N}
\end{array}
$$

Expanding all terms in the above equation

$$
\begin{aligned}
& \mathcal{C}_{t}=\left(x_{s}\left(-V_{2 x} \cdot y_{s}+V_{2 y} \cdot x_{s}\right)-y_{s}\left(-V_{1 x} \cdot y_{s}+V_{1 y} \cdot x_{s}\right)+\right. \\
& \left.\left\langle\left(\begin{array}{c}
-V_{2 y} \cdot y_{s}-V_{2 x} \cdot x_{s} \\
V_{1 y} \cdot y_{s}+V_{1 x} \cdot x_{s}
\end{array}\right), \mathcal{N}\right\rangle\right) \mathcal{N} \\
& =\left(-V_{2 x} \cdot x_{s} \cdot y_{s}+V_{2 y} \cdot x_{s}^{2}+V_{1 x} \cdot y_{s}^{2}-V_{1 y} \cdot x_{s} \cdot y_{s}\right. \\
& \left.+V_{2 y} \cdot y_{s}^{2}+V_{2 x} . x_{s} \cdot y_{s}+V_{1 y} . x_{s} \cdot y_{s}+V_{1 x} . x_{s}^{2}\right) \mathcal{N} \\
& =\left(V_{1_{x}}\left(x_{s}{ }^{2}+y_{s}^{2}\right)+V_{2 y}\left(x_{s}^{2}+y_{s}^{2}\right)\right) \mathcal{N} \\
& =\left(V_{1_{x}}+V_{2 y}\right) \mathcal{N}=\operatorname{div}(\mathcal{V}) \mathcal{N}
\end{aligned}
$$

As a corollary to Theorem 1, we have 
Corollary 1 The direction in which the circulation of of the vector field $\mathcal{V}$ along the curve $\mathcal{C}$ is increasing most rapidly is given by $\frac{\partial \mathcal{C}}{\partial t}=\operatorname{div}\left(\mathcal{V}^{\perp}\right) \mathcal{N}$.

Proof: Using the properties of scalar products in Eq. (2)

$$
\begin{aligned}
\operatorname{Circ}(t) & =\int_{0}^{L(t)}\langle\mathcal{V}, \mathcal{T}\rangle d s \\
& =\int_{0}^{L(t)}\left\langle\mathcal{V}^{\perp}, \mathcal{T}^{\perp}\right\rangle d s \\
& =\int_{0}^{L(t)}\left\langle\mathcal{V}^{\perp}, \mathcal{N}\right\rangle d s
\end{aligned}
$$

Hence the circulation of the vector field $\mathcal{V}$ along the curve is just the inward flux of the vector field $\mathcal{V}^{\perp}$ through it and the result follows from Theorem 1.

\section{Volumetric Extensions}

We now consider the volumetric extension of the flux maximizing flow. In order to do this, we will need to set up some notation. Let $\mathcal{S}:[0,1] \times[0,1] \rightarrow \mathcal{R}^{3}$ denote a compact embedded surface with (local) coordinates $(u, v)$. Let $\mathcal{N}$ be the inward unit normal. We set

$$
\mathcal{S}_{u}:=\frac{\partial \mathcal{S}}{\partial u}, \quad \mathcal{S}_{v}:=\frac{\partial \mathcal{S}}{\partial v} .
$$

Then the infinitesimal area on $\mathcal{S}$ is given by

$$
d \mathcal{S}=\left(\left\|\mathcal{S}_{u}\right\|^{2}\left\|\mathcal{S}_{v}\right\|^{2}-\left\langle\mathcal{S}_{u}, \mathcal{S}_{v}\right\rangle^{2}\right)^{1 / 2} d u d v .
$$

Let $\mathcal{V}=\left(V_{1}(x, y, z), V_{2}(x, y, z), V_{3}(x, y, z)\right)$ be a vector field defined for each point $(x, y, z)$ in $\mathcal{R}^{3}$. The total inward flux of the vector field through the surface is defined in a manner analogous to the 2D case and is given by the surface integral

$$
\operatorname{Flux}(t)=\int_{0}^{1} \int_{0}^{1}\langle\mathcal{V}, \mathcal{N}\rangle d \mathcal{S} .
$$

However, an analogous definition does not exist for the circulation because the notion of a tangent in 2D is now replaced by that of a tangential plane ${ }^{1}$. It should not come as a surprise that the earlier result generalizes to 3D.

Theorem 2 The direction in which the inward flux of the vector field $\mathcal{V}$ through the surface $\mathcal{S}$ is increasing most rapidly is given by $\frac{\partial \mathcal{S}}{\partial t}=\operatorname{div}(\mathcal{V}) \mathcal{N}$.

Sketch of Proof: The proof is more involved than the 2D case, and will be presented in its entirety in separate work. As before, the idea is to calculate the first variation of the flux functional with respect to $t$ :

$$
\operatorname{Flux}^{\prime}(t)=\underbrace{\int_{0}^{1} \int_{0}^{1}\left\langle\mathcal{V}_{t}, \mathcal{N}\right\rangle d \mathcal{S}}_{I_{1}}+\underbrace{\int_{0}^{1} \int_{0}^{1}\left\langle\mathcal{V}, \mathcal{N}_{t}\right\rangle d \mathcal{S}}_{I_{2}}
$$

\footnotetext{
${ }^{1}$ In fluid mechanics one defines the circulation along a chosen $3 \mathrm{D}$ closed curve on the surface, but this is not a surface integral.
}

It is straightforward to show that $I_{1}$ has the desired form of an inner product of $\mathcal{S}_{t}$ with another vector. $I_{2}$ can be simplified and combined with $I_{1}$ using integration by parts, the chain rule and properties of cross products. The components of the flow in the tangential plane to the surface at each point can then be dropped, since these affect only the parametrization, yielding the result

$$
\mathcal{S}_{t}=\operatorname{div}(\mathcal{V}) \mathcal{N} .
$$

\section{Blood Vessel Segmentation}

We now tailor the flux maximizing flow to the segmentation of blood vessels in MRA images. We begin by reviewing a few recent approaches to this problem. Wilson and Noble have introduced a Gaussian mixture model to characterize the physical properties of blood flow [19]. Krissian et al. propose a method which incorporates a Gaussian model for the intensity distribution as a function of distance from vessel centerlines, and exploits properties of the Hessian to obtain geometric estimates [11]. Koller et al. have introduced a multi-scale method for the detection of curvilinear structures in 2D and 3D data [10] which combines the responses of steerable linear filters and also exploits the Hessian matrix to obtain geometric estimates. Bullitt et al. have introduced a method for obtaining 3D vascular trees which calculates vessel centerlines as intensity ridges in the data [2]. Several of the above approaches require second derivative computations, hence they are often used in conjunction with vessel-preserving smoothing techniques [6].

Whereas the potential of several of the above approaches has been demonstrated, their ability to recover low contrast thin vessels remains unclear. A recent framework which has been developed with this as one of its goals is the work of Lorigo et al. [12]. The main idea is to regularize a geometric flow in $3 \mathrm{D}$ using the curvature of a $3 \mathrm{D}$ curve, rather than the classical mean curvature based regularizations which tend to annihilate thin structures. This approach is based on the recent level set theory developed for mean curvature flows in arbitrary co-dimension [1]. The flow is given by

$$
\psi_{t}=\lambda\left(\nabla \psi, \nabla^{2} \psi\right)+\frac{g^{\prime}}{g} \nabla \psi \cdot \mathbf{H} \frac{\nabla \mathbf{I}}{\|\nabla \mathbf{I}\|}
$$

and has a variational interpretation as the gradient flow that minimizes a weighted curvature functional. Here $\psi$ is an embedding surface whose zero level set is the evolving 3D curve, $\lambda$ is the smaller nonzero eigen value of a particular matrix [1] $g$ is an image-dependent weighting factor, $\mathbf{I}$ is the intensity image and $\mathbf{H}$ is its Hessian. In order to attract the flow to blood vessel boundaries, the second term of the equation is multiplied by a (heuristic) factor $\rho(\nabla \psi \cdot \nabla \mathbf{I})$, where $\rho$ is a constant. This favours level sets whose normals are locally aligned to the direction of image intensity gradients, but the flow then loses its pure variational interpretation. 


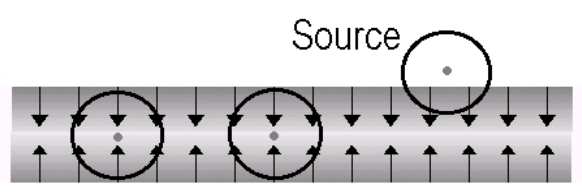

Sinks

Figure 1: An illustration of the gradient vector field in the vicinity of a blood vessel. Assuming a uniform background intensity, at its centerline, at the scale of the vessel's width, the total outward flux is negative. Outside the vessel, at a smaller scale, the total outward flux is positive.

\subsection{The Flux Maximizing Flow}

In order to adapt the flux maximizing flow to blood vessel segmentation, we shall consider the gradient $\nabla \mathbf{I}$ of the original intensity image $\mathbf{I}$ to be the vector field $\mathcal{V}$ whose inward flux through the evolving curve (or surface) is maximized. An important consideration in the implementation of Eq. (3) is that since the divergence of the vector field needs to be calculated, implicitly second derivatives of $\mathbf{I}$ are being used. However, the numerical computation can be made much more robust by exploiting a consequence of the divergence theorem. The divergence at a point is defined as the net outward flux per unit area, as the area about the point shrinks to zero. Via the divergence theorem,

$$
\int_{\Delta a} \operatorname{div}(\mathcal{V}) \mathrm{da} \equiv \int_{\mathrm{L}}<\mathcal{V}, \mathcal{N}>\mathrm{dl}
$$

Here $\Delta a$ is the area, $L$ is its bounding contour and $\mathcal{N}$ is the outward normal at each point on the contour. The formulation extends to 3D by replacing the contour integral with a surface integral.

For our numerical implementations we use this flux formulation along the boundaries of circles (in 2D) or spheres (in 3D) of varying radii, corresponding to a range of blood vessel widths. The chosen flux value at a particular location is the maximum (magnitude) flux over the range of radii. In contrast to other multi-scale approaches where combining information across scales is non-trivial [11] normalization across scales is straightforward in our case. One simply has to divide by the number of entries in the discrete sum that approximates Eq. (6). Locations where the total outward flux (which is proportional to the divergence) is negative correspond to sinks; locations where the total outward flux is positive correspond to sources, as illustrated in Figure 1. Hence, the flux maximizing flow in Eq (3) has the desirable effect that, when seeds are placed within blood vessels, the sources outside boundaries prevent the flow from leaking.

In order to implement the flow, we use the level set representation for curves flowing according to functions of curvature [14]. Let $\mathcal{C}(p, t): S^{1} \times[0, \tau) \rightarrow \mathbf{R}^{2}$ be a family of

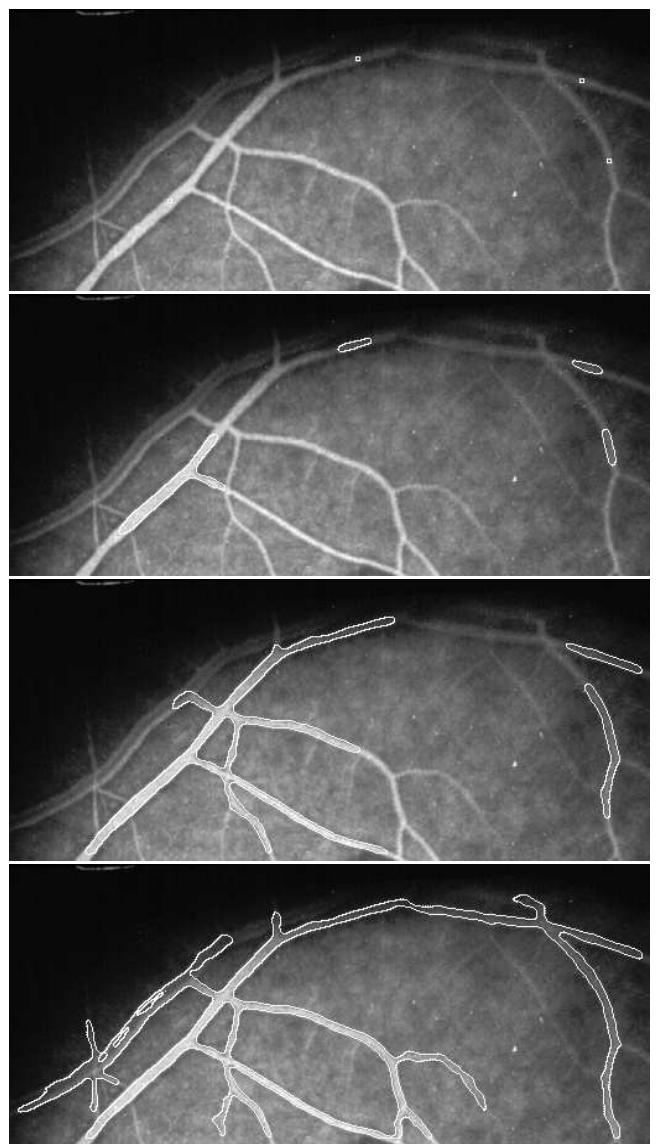

Figure 2: An illustration of the flux maximizing flow for a portion of a $2 \mathrm{D}$ retinal angiography image. The evolution of a few isolated seeds is superimposed on the original. These follow the direction of shading to reconstruct the blood vessel boundaries. Observe that the very low contrast vessel at the top is successfully reconstructed.

curves satisfying the curve evolution equation

$$
\mathcal{C}_{t}=F \mathcal{N},
$$

where $F$ is an arbitrary (local) scalar speed function. Then it can be shown that if $\mathcal{C}(p, t)$ is represented by the zero level set of a smooth and Lipschitz continuous function $\Psi: \mathbf{R}^{2} \times[0, \tau) \rightarrow \mathbf{R}$, the evolving surface satisfies $\Psi_{t}=F\|\nabla \Psi\|$, which is solved using a combination of straightforward discretization and numerical techniques derived from hyperbolic conservation laws [14]. For hyperbolic terms, care must be taken to implement derivatives with upwinding in the proper direction. The evolving curve $\mathcal{C}$ is then obtained as the zero level set of $\Psi$. The formulation is analogous for the case of surfaces evolving in 3D.

\section{Examples}

We now illustrate the flux maximizing flow with simulations implemented in a level-set framework using narrow 
banding for computational efficiency. Figure 2 shows the flow on a portion of a $2 \mathrm{D}$ retinal angiogram ${ }^{2}$. Notice how a few seeds evolve along the direction of shading (orthogonal to the image intensity gradient direction) to reconstruct thin or low contrast structures, e.g., the top portion of Figure 2. Most other flows, particularly ones with a constant inflation term, would leak through such boundaries. The introduction of a curvature-based regularization term may prevent leaking to an extent, but the flow would then be halted at narrow regions as well, since the curvature term would dominate and would push the evolving curve back.

Figure 3 illustrates the flow on a portion of a 3D MRA image of blood vessels in the head. A maximal intensity projection of the data is shown on the top left, followed by the evolution of a few seeds initialized uniformly in regions of high inward flux, which is similar to the idea of using 'bubbles' [18]. Notice how the seeds elongate in the direction of blood vessels, which is once again the evolution we expect since it maximizes the rate of increase of inward flux through them. The effectiveness of the flow in reconstructing the full data set is illustrated in Figure 4. The main blood vessels, which have the higher inward flux, are the first to be captured.

\section{Conclusion and Discussion}

The main contribution of this paper is the formulation and derivation of a flux maximizing flow and its adaptation to blood vessel segmentation. The derivation leads to the simple interpretation that each point on a curve (2D) or surface (3D) should move in the inward normal direction, by an amount proportional to the divergence of the auxiliary vector field.

We chose to initialize the flow by placing seeds in regions of high inward flux to illustrate its properties. However, one might consider thresholding the flux to obtain an initialization which has already reconstructed large portions of blood vessels. In practice we have found that this performs better than simple thresholding of the original data as in $[19,12]$. However, more work remains to be done in order to validate this flow against ground truth or expert segmentations on a diverse range of images. We also chose not to introduce a regularization term in the variational formulation; whether this can be incorporated in the derivation remains to be investigated. The flow could also smoothed after it has converged using the geometric heat equation or its affine invariant version in 2D, or the co-dimension 2 flow in $3 \mathrm{D}$ [12].

\footnotetext{
${ }^{2}$ This is a portion of a gray level image which was obtained from a web page. A considerable amount of numerical precision was lost since the image was saved with only 256 gray levels. Hence, it serves as a good test case for the flow.
}

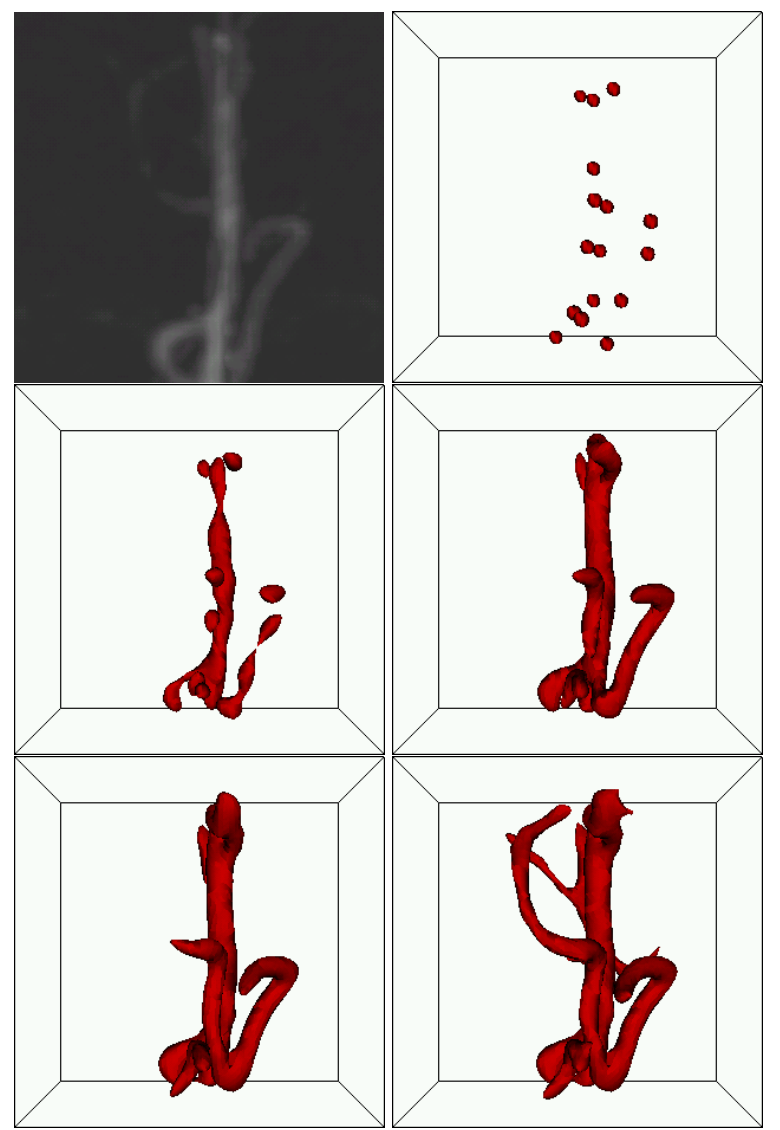

Figure 3: An illustration of the flux maximizing flow for a portion of a 3D MRA image of blood vessels in the head. A maximum-intensity projection of the cropped portion is shown on the top left and the other images depict the evolution of a few isolated seeds according to the flux maximizing flow. The full reconstruction is shown in Figure 4.

Acknowledgements This work was supported by grants from CFI, FCAR and NSERC. We thank Terry Peters and Bruce Pike for the MRA data.

\section{References}

[1] L. Ambrosio and H. M. Soner. Level set approach to mean curvature flow in arbitrary codimension. Journal of Differential Geometry, 43:693-737, 1996.

[2] E. Bullitt, S. Aylward, A. Liu, J. Stone, S. K. Mukherjee, C. Coffey, G. Gerig, and S. M. Pizer. 3d graph description of the intracerebral vasculature from segmented mra and tests of accuracy by comparison with x-ray angiograms. In IPMI'99, pages 308-321, 1999.

[3] V. Caselles, F. Catte, T. Coll, and F. Dibos. A geometric model for active contours in image processing. Numerische Mathematik, 66:1-31, 1993.

[4] V. Caselles, R. Kimmel, and G. Sapiro. Geodesic active contours. In ICCV'95, pages 694-699, 1995.

[5] T. Chan and L. Vese. An efficient variational multiphase motion for the mumford-shah segmentation model. In Asilomar Conference on Signals and Systems, October 2000. 


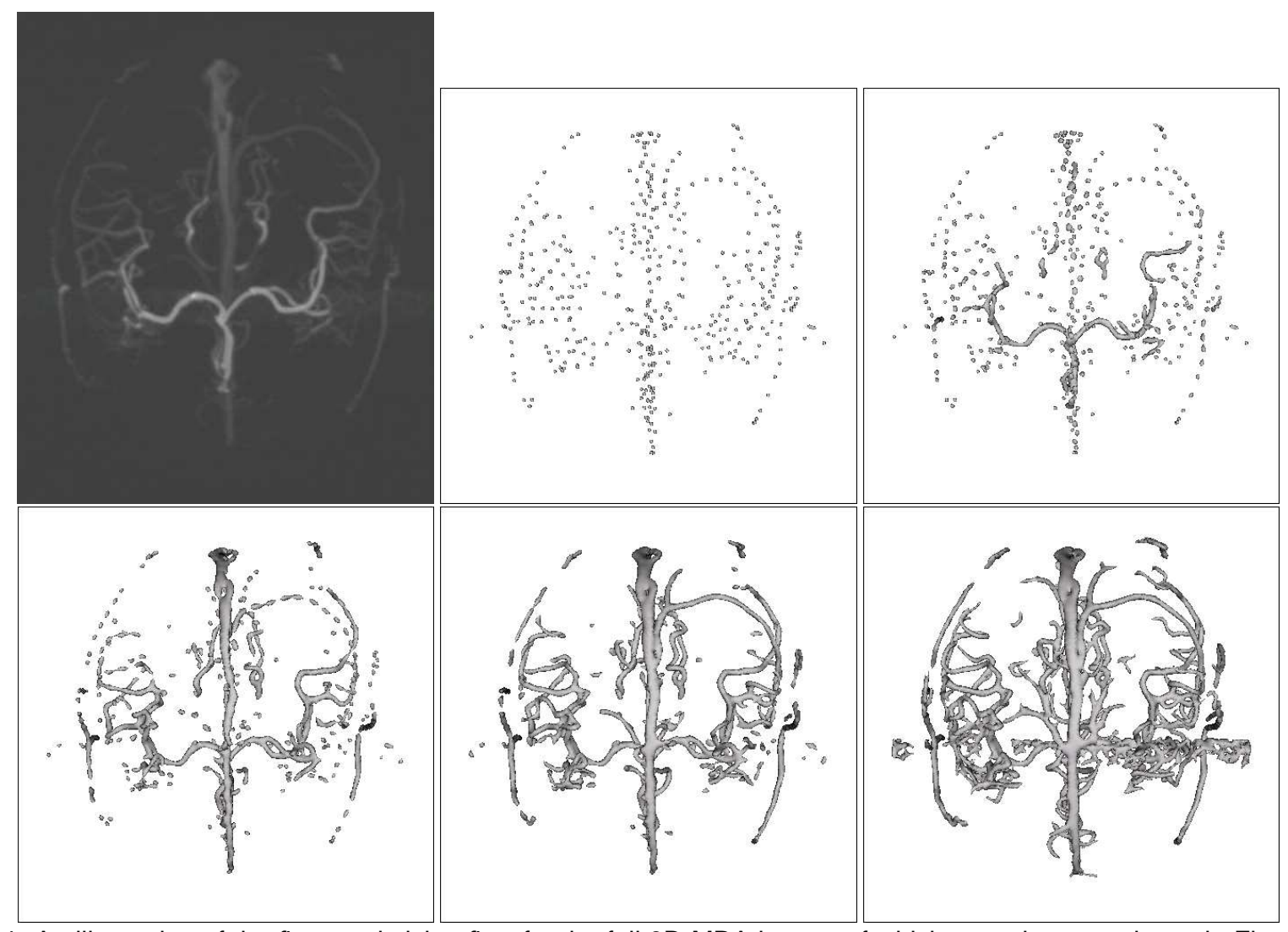

Figure 4: An illustration of the flux maximizing flow for the full 3D MRA image, of which a portion was shown in Figure 3. A maximum-intensity projection of the data is shown on the top left and the other images depict the evolution of a few isolated seeds. The main vessels, which have higher inward flux, are the first to be reconstructed.

[6] A. Frangi, W. Niessen, K. L. Vincken, and M. A. Viergever. Multiscale vessel enhancement filtering. In MICCAI'98, pages 130-137, 1998.

[7] M. Kass, A. Witkin, and D. Terzopoulos. Snakes: Active contour models. International Journal of Computer Vision, 1:321-331, 1987.

[8] S. Kichenassamy, A. Kumar, P. Olver, A. Tannenbaum, and A. Yezzi. Gradient flows and geometric active contour models. In ICCV'95, pages 810-815, 1995.

[9] B. B. Kimia, A. Tannenbaum, and S. W. Zucker. Toward a computational theory of shape: An overview. Lecture Notes in Computer Science, 427:402-407, 1990.

[10] T. M. Koller, G. Gerig, G. Székely, and D. Dettwiler. Multiscale detection of curvilinear structures in 2-d and 3-d image data. In ICCV'95, pages 864-869, 1995.

[11] K. Krissian, G. Malandain, N. Ayache, R. Vaillant, and Y. Trousset. Model-based multiscale detection of $3 \mathrm{~d}$ vessels. In CVPR'98, pages 722-727, 1998.

[12] L. M. Lorigo, O. Faugeras, E. L. Grimson, R. Keriven, R. Kikinis, A. Nabavi, and C.-F. Westin. Codimensiontwo geodesic active contours for the segmentation of tubular structures. In CVPR'2000, volume 1, pages 444-451, 2000.

[13] R. Malladi, J. A. Sethian, and B. C. Vemuri. Shape modeling with front propagation: A level set approach. IEEE Transactions on Pattern Analysis and Machine Intelligence, 17(2):158-175, February 1995.
[14] S. J. Osher and J. A. Sethian. Fronts propagating with curvature dependent speed: Algorithms based on hamilton-jacobi formulations. Journal of Computational Physics, 79:12-49, 1988.

[15] N. Paragios and R. Deriche. Geodesic active regions for supervised texture segmentation. In ICCV'99, pages 926-932, September 1999.

[16] J. Shah. Recovery of shapes by evolution of zero-crossings. Technical report, Dept. of Mathematics, Northeastern University, Boston, MA, 1995.

[17] K. Siddiqi, Y. B. Lauzière, A. Tannenbaum, and S. W. Zucker. Area and length minimizing flows for shape segmentation. IEEE Transactions on Image Processing, 7(3):433443, 1998.

[18] H. Tek and B. B. Kimia. Volumetric segmentation of medical images by three-dimensional bubbles. Computer Vision and Image Understanding, 65(2):246-258, 1997.

[19] D. L. Wilson and A. Noble. Segmentation of cerebral vessels and aneurysms from $\mathrm{mr}$ aniography data. In IPMI'97, pages 423-428, 1997.

[20] C. Xu and J. Prince. Snakes, shapes and gradient vector flow. IEEE Transactions on Image Processing, 7(3):359369, 1998.

[21] A. Yezzi, A. Tsai, and A. Willsky. A statistical approach to snakes for bimodal and trimodal imagery. In ICCV'99, pages 898-903, September 1999. 